wound healing due to immunosuppression. Krambeck et al. retrospectively reviewed all cases of allograft nephrolithiasis treated with percutaneous nephrolithotomy at the Mayo Clinic, Rochester, MN, during the period June 1988 to February 2006, in order to determine the long-term safety and efficacy of the procedure in this setting.

A total of 13 patients were analyzed; the most common presenting symptom was acute kidney injury (46.1\% of patients) and the most common stone composition was calcium oxalate monohydrate $(46.2 \%$ of patients). No adjacent organ injury or other intraoperative complications occurred during percutaneous nephrolithotomy, although $3(23.1 \%)$ patients developed manageable complications immediately after the procedure, including sepsis and gastrointestinal bleeding; these events were attributed primarily to immunosuppression. Percutaneous nephrolithotomy rendered $10(76.9 \%)$ patients stone-free, as indicated by nephrostography $24 \mathrm{~h}$ following the procedure. The remaining $3(23.1 \%)$ patients became stone-free after nephrolithotomy was repeated.

The mean serum creatinine level and mean Cockcroft-Gault-estimated creatinine clearance remained stable during followup (average 5.3 years, range $0.6-9$ years), although one patient, who had renal dysfunction before nephrolithotomy, required a second renal transplant 1 year later. The rate of stone recurrence was $7.7 \%$ (1 patient).

This small retrospective study suggests that percutaneous nephrolithotomy is an effective treatment for nephrolithiasis in kidney transplants and is not associated with long-term morbidity.

Original article Krambeck AE et al. (2008) Percutaneous nephrolithotomy success in the transplant kidney. J Urol 180: 2545-2549

\section{Combined transplantation protects against graft rejection}

Liver allografts have been known for some time to protect against organ rejection when simultaneously transplanted with other allografts. Rana et al. have now shown that heart and kidney allografts are also immunoprotective and are themselves protected from rejection when transplanted simultaneously with another organ.

The authors searched the United Network of Organ Sharing database and extracted followup data for all thoracic, kidney, intestine and liver transplant recipients over 18 years of age who had undergone transplantation within a 10-year period. In total, data from 133,416 patients who were followed up for a mean of 3.13 years were examined.

In patients who underwent combined heartkidney transplantation or combined liver-kidney transplantation, the incidence of rejection of any allograft at 1 year was lower and the rate of rejection-free survival higher than in patients who received a single allograft. In addition, the renal rejection rate in patients who underwent heart transplantation then received a kidney allograft from a different donor at a later date remained lower than that in patients who underwent kidney transplantation only. Simultaneous intestine or pancreas transplantation did not reduce rejection rates or improve rejection-free survival in kidney or liver allograft recipients. Patients who received double lung transplants or double kidney transplants showed a lower rejection rate and greater rejection-free survival than patients who received single organ transplants.

The authors suggest that combined organ allografts modulate the immune system favorably for one or both allografts.

Original article Rana A et al. (2008) The combined organ effect: protection against rejection? Ann Surg 248: 871-879 